\title{
Liver transplantation in pediatric patients with progressive familial intrahepatic cholestasis: single center experience of seven cases
}

\author{
Shin Hwang, Jung-Man Namgoong, Kyung Mo Kim, Seak Hee Oh, Seung-Mo Hong
}

Department of Surgery, Asan Medical Center, University of Ulsan College of Medicine, Seoul, Korea

Background: Progressive familial intrahepatic cholestasis (PFIC) is an autosomal recessive inherited disease requiring liver transplantation (LT). The objective of this study was to investigate the clinicopathological features and posttransplant courses of seven LT recipients with PFIC.

Methods: This was a retrospective single-center study of patients with PFIC who underwent LT from January 2013 to June 2020. Results: Two and five patients were diagnosed with PFIC type 1 and type 2, respectively. For all seven patients, age at PFIC onset was at birth. Jaundice was present in all cases. Mean pretransplant total and direct bilirubin levels were $16.1 \pm 8.1 \mathrm{mg} / \mathrm{dL}$ and $12.4 \pm 6.2 \mathrm{mg} / \mathrm{dL}$, respectively. Median patient age and body weight at LT were 10 months and $7 \mathrm{~kg}$, respectively. Types of donors were mothers of patients in four and deceased donors in three. All five patients with PFIC type 2 recovered uneventfully. Each patient with PFIC type 1 underwent retransplantation due to graft failure or died due to multi-organ failure. Overall graft and patient survival rates at 5 years were $66.7 \%$ and $83.3 \%$, respectively. Bile salt export pump immunohistochemical staining showed normal canalicular expression in two patients with PFIC type 1, focal loss in two patients with PFIC type 2, and total loss in three patients with PFIC type 2.

Conclusions: LT is currently the only effective treatment for PFIC-associated end-stage liver diseases. It is mandatory to perform regular follow-up due to the risk of complications including steatohepatitis, especially for patients with PFIC type 1.

Corresponding author: Shin Hwang

E-mail: shwang@amc.seoul.kr

(c) The Korean Society for Transplantation

This is an Open Access article distributed under the terms of the Creative Commons Attribution Non-Commercial License (http://creativecommons.org/licenses/by-nc/4.0/) which permits unrestricted non-commercial use, distribution, and reproduction in any medium, provided the original work is properly cited. 